\title{
zo \\ El racismo de Estado y el estado de excepción como respuesta neoliberal a las crisis humanitarias del siglo XXI. Biopoder y biopolítica como una crítica al Estado-nación
}

\author{
Moisés Gómez \\ Universidad "José Simeón Cañas", UCA
}

\section{Introducción}

Vivimos en mundo convulsio- humanos, todo lo hace la planta nado, de manera muy clara podemos identificar varias crisis globales: la primera, es la crisis ambiental, el capitalismo ha ocupado y depredado toda la Tierra, el planeta y sus recursos están desequilibrados, el modo de producción capitalista exige a la Tierra más de lo que ella puede por sí misma regenerar. La segunda crisis es la del trabajo, el capitalismo prescinde, gracias al desarrollo tecnológico, del trabajador. El aparato productivo funciona mejor y produce más sin trabajadores de producción automatizada. $\mathrm{El}$ desempleo estructural es lo que hay, millones de personas sin acceso a un empleo decente. El capitalista de hoy logra más ganancias explotando la maquinaria en vez de obtener ganancias por la vía de explotación del ser humano. Y eso no está mal, el problema es que el control de la producción y sus réditos está concentrado en muy pocas manos. Las consecuencias de la creación de riqueza sin la intervención humana y el desplazamiento del hombre por 
la máquina todavía no lo podemos dimensionar.

Por último,el mundo está viviendo la peor crisis humanitaria de toda su historia, una pequeña muestra es la situación de los refugiados desde la Segunda Guerra Mundial. Más de 65 millones de personas se han visto obligados a abandonar sus hogares debido a terribles conflictos, a la violencia y la persecución. El triángulo norte no escapa a esta tragedia humanitaria. El crimen organizado sigue alimentando la violencia en la región (principalmente maras, pandillas y carteles de la droga). Al menos 711,500 personas se encuentran desplazadas internamente en Honduras, El Salvador y Guatemala y cientos de miles de personas están huyendo de las zonas violentas hacia otros países como parte de los flujos mixtos de migración. En cuanto a deportaciones de los países de destino, en 2015, fueron deportadas 234,561 personas de Estados Unidos y México, lo que demuestra que la situación es crítica porque "alrededor del $35 \%$ de los deportados son personas en necesidad de protección internacional, que han huido por la violencia" tal como sostiene el Consejo Noruego para Refugiados (Consejo Noruego para Refugiados Violencia generalizada en TNCA: Reporte IDMC 2016).
El mundo en crisis es nuestro punto de partida y nuestro análisis propone que lo anterior es una de las consecuencias de medidas políticas y sociales a partir del racismo de Estado y del Estado de Excepción. El racismo de Estado fue desarrollado en el texto Defender la sociedad por Michel Foucault en las clases del año 1975-76, mientras que la noción de estado de excepción lo desarrolló, entre otros, el italiano Giorgio Agamben. Los dos conceptos resumen lo que llamo respuesta neoliberal a las distintas crisis de la humanidad contemporánea.

La relación de poder entre aquellos que deciden la política socioeconómica y los que la sufren es asimétrica. ¿Cómo quedan los derechos humanos de estas mayorías afectadas? Mal, quedan mal. Prácticamente, los derechos humanos de las grandes poblaciones que huyen de las violencias están entre paréntesis o por lo menos suspendidos hasta nuevo aviso. Los derechos humanos se realizan teniendo como sujeto al libre mercado cuyo reverso es el sujeto propietario, calculador y consumidor. Los derechos humanos son apropiados por el sujeto consumidor de alto nivel. ¿Cómo llegamos a esta respuesta neoliberal? 


\title{
1. El racismo de Estado como mecanismo de defensa de la sociedad en el Estado Moderno
}

En el mercado libre es natural la victoria del fuerte y legítima la aniquilación del débil. Así se eleva el racismo a la categoría de doctrina económica. (Galeano, 2010)

Con frecuencia leemos notas de prensa con información similar a esta:

Más de 3 mil 400 migrantes han muerto o desaparecido en lo que va del año (2016) al tratar de cruzar fronteras en distintas partes del mundo, el $80 \%$ de ellos al intentar el cruce del mar para llegar a Europa, dijo el miércoles la Organización Internacional para las Migraciones. (La Jornada, 15 de junio 2016)

Los muertos sin visa no importan, biopoder. De la mano del avance en no son turistas, ni inversionistas, los casi 4 mil muertos en el intento de cruzar fronteras. Son pobladores fuera de su lugar de nacimiento, son gente empobrecida por sus corruptos dirigentes políticos. ¿Qué lógicas subyacen en la comprensión de esta realidad?

El análisis del racismo de Estado implica comprender la noción de lítica):

La disciplina es el poder cuyo objeto es el individuo (anatomopo-

\begin{abstract}
A partir del siglo XVIII aparecen las técnicas del poder que se centraban esencialmente en el cuerpo, el cuerpo individual [...] se trataba también de las técnicas por las que esos cuerpos quedaban bajo supervisión y se intentaba incrementar su fuerza útil mediante el ejercicio, el adiestramiento [...] toda la tecnología que podemos llamar tecnología disciplinaria del trabajo. (Foucault, 2010, 2010, p. 207)
\end{abstract}

La nueva gubernamentalidad como poder se ocupará entonces de lo siguiente:
1) De la proporción de nacimientos, de decesos, de las tasas de reproducción, de la fecundidad de 
la población; en una palabra, de la demografía.
2) De las enfermedades endémicas: de la naturaleza, de la extensión, de la duración, de la intensidad de las enfermedades reinantes en la población, de la higiene pública.

a partir del siglo XVIII, se buscó racionalizar los problemas planteados a la práctica gubernamental por los fenómenos propios de un conjunto de vivientes en cuanto población: salud, higiene, natalidad, longevidad, raza. (Foucault, 1994a, p. 818)

3) De la vejez, de las enfermedades que dejan al individuo fuera del mercado del trabajo; también, entonces, de los seguros individuales y colectivos, de la jubilación.

4) De las relaciones con el medio geográfico, con el clima, el urbanismo y la ecología.
Las poblaciones en movimiento a la luz de la disciplina moderna son poblaciones indisciplinadas, violentan el orden que indica que ellas pertenecen a un territorio económico específico y concreto y donde el dispositivo disciplinario se propone:

Hacer útil la pobreza fijándola en un aparato de producción, y, en el peor de los casos aligerar al máximo el peso que representa para el resto de la sociedad. Cómo hacer trabajar a los pobres "útiles" cómo transformarlos en mano de obra rentable. Se perfila así toda una analítica utilitaria de la pobreza en la que comienza a aparecer el problema específico de la enfermedad de los pobres en relación a los imperativos del trabajo y a la necesidad de la producción. (Foucault, 1991, p. 93)

Sin embargo, como pueblo - no como población - su movimiento migratorio rompe esa disciplina estacionaria, fija y que trata de sujetarlo.
La biopolítica busca un tipo de organización social que permita el enriquecimiento en la lógica del mercado: 
La primera de las grandes operaciones de la disciplina es la constitución de cuadros vivos que transforma las multitudes confusas inútiles o peligrosas, en multiplicidades ordenadas. La constitución de cuadros ha sido uno de los grandes problemas de la tecnología científica, política y económica del siglo XVIII: observar, controlar, regularizar la circulación de las mercancías y de la moneda y construir así un cuadro económico que pueda valer como principio de enriquecimiento; inspeccionar a los hombres, comprobar su presencia y su ausencia, y constituir un registro general [...] El cuadro en el siglo XVIII es a la vez una técnica de poder y un procedimiento de saber. Se trata de organizar lo múltiple, de procurarse un instrumento para recorrerlo y dominarlo, se trata de imponerle un orden. (Foucault, 1978, p. 152)

El orden y la disciplina ${ }^{11}$ de los que se habla son el orden y la disciplina del mercado como norma, que se expresa como ley de la competencia, de la lucha por los recursos escasos en la que el mercado, y no el gobierno, asigna mejor los recursos. De esto vamos a tratar más adelante.

Este orden se logra por medio del proceso de "normalización" tanto del cuerpo individual así como del cuerpo-población, de aquí las sociedades modernas no son simplemente sociedades de disciplinarización, sino de normalización:

De una manera aún más general, puede decirse que el elemento que va a circular de lo disciplinario a lo regularizador, que va a aplicarse del mismo modo al cuerpo y a la población, que permite controlar el orden disciplinario del cuerpo y los acontecimientos aleatorios de una multiplicidad biológica, el elemento que circula de una a la otra, es la norma. La norma es lo que puede aplicarse tanto a un cuerpo al que se quiere disciplinar como a una población a que se pretende regularizar [...] La sociedad de normalización es una sociedad donde se cruzan, según una articulación ortogonal, la norma de la disciplina y la norma de la regulación [...] estamos en un poder que se hizo cargo del cuerpo y de la vida. (Foucault, 2010, p. 217)

Como sabemos, la norma es lo genérico, prescribe conductas, no siempre la norma coincide con la ley, pero en este caso de Foucault $y$ en el contexto del liberalismo y neoliberalismo, la norma coincide con la ley, entendida como la ley del mercado. La norma que cruza el 
cuerpo individual y a la población es la ley del mercado. Por eso es que en otro lugar ha dicho Foucault que el hombre moderno es un animal en cuya política está en cuestión su ser viviente (Foucault, 1994a, p. 827).
¿Cómo entra el racismo en el Estado regido por la biopolítica? Tradicionalmente se ha considerado que la soberanía y el soberano muestran su poder en la decisión de hacer morir. Sucede un cambio radical en el Estado Moderno, el poder soberano no se expresa en el ejercicio de matar sino que es todo lo opuesto:

\begin{abstract}
[...] el poder es cada vez menos el derecho de hacer morir y cada vez más el derecho de intervenir para hacer vivir, y de intervenir sobre la manera de vivir, y sobre el 'cómo' de la vida; a partir de este momento, entonces, en que el poder interviene, sobre todo en este nivel, para ampliar la vida, para controlar los accidentes, lo aleatorio, las deficiencias, de golpe la muerte, como término de la vida, es evidentemente el término, el límite, el extremo del poder. (Foucault, 2010, p. 212)
\end{abstract}

Para Foucault, el Estado Moderno gestionado biopolíticamente realza la vida humana en sus principales aspectos, desarrollo científico y política se conectan para obtener una sociedad saludablemente en buen estado.

Se pregunta Foucault, ¿cómo es que el Estado Moderno, en tanto que institución racional, ejerce el antiguo derecho del soberano de "dejar morir"? Tampoco es que el Estado Moderno haya renunciado al ejercicio soberano, pero, ¿cómo hacerlo si este Estado tiene como objetivo esencial ejercer el poder para "hacer vivir"? La salida no es otra que recurrir al racismo ${ }^{3}$ de Estado como mecanismo que le permite ejercer el derecho de matar:

La raza, el racismo son la condición que hace aceptable dar muerte en una sociedad de normalización. Donde hay una sociedad de normalización, donde existe un biopoder, el racismo es indispensable como condición para poder dar muerte a alguien, para poder dar muerte a los otros. En la medida en que el Estado funciona en la modalidad del biopoder, su función mortífera solo puede ser asegurada por el racismo. (Foucault, 2010, p. 219) 
Las colonizaciones, las guerras del mundo que entran en conflictos contemporáneas son un ejemplo armados donde la eliminación del de ese racismo, de hecho, la gran otro es lo primordial gracias a la mayoría de nuestros refugiados ayuda de las técnicas y tecnologías actualmente provienen de zonas en del poder: guerra, cada vez vemos más zonas

Un poder que tiene derecho de vida y muerte y quiere funcionar con los instrumentos, los mecanismos y la tecnología de la normalización, también es preciso que pase por el racismo. Desde luego, cuando hablo de dar muerte no me refiero simplemente al asesinato directo, sino también a todo lo que puede ser asesinato indirecto: el hecho de exponer a muerte, multiplicar el riesgo de muerte de algunos, o sencillamente, la muerte política, la expulsión, el rechazo. (Foucault, 2010, p. 220.)

\subsection{Del racismo de Estado al racismo económico}

Para Foucault, no se puede biopolítica se dedica al análisis del comprender el nacimiento de la nuevo arte de gobernar en la que la biopolítica sin atender el liberalismo regulación del sistema ya no es de y su evolución en el neoliberalismo. derecho sino de hecho: En toda su obra, El nacimiento de la

Decir que es una limitación de hecho significa que si el gobierno llega alguna vez a atropellarla, a traspasar las fronteras que se le imponen, no será pese a ello ilegítimo, no habrá desertado, en cierto modo, de su propia esencia, no se verá desposeído de sus derechos fundamentales. Decir que hay una limitación de hecho de la práctica gubernamental querrá decir que el gobierno que desconozca esa limitación será simplemente un gobierno, insistamos, no ilegítimo, no usurpador, sino torpe, inadaptado, un gobierno que no hace lo que conviene. (Foucault, 2012, p. 23)

Al respecto de lo anterior conviene citar la pertinente afirmación de Hinkelammert (2007), "no se escoge lo que sea. Lo es, aunque no lo queramos" (p. 76). Esto es la sociedad organizada y respetuosa de la propiedad privada y del contrato, gobierno que no respeta esto es torpe y no hace lo que conviene.

Por otra parte, el principio de limitación del gobierno ya no es extrín- 
seco sino intrínseco de tal manera que la práctica gubernamental tiene que hacerse cargo de la biopolítica (hacer vivir la población) en una situación donde el mismo gobierno debe autoimponerse ciertas reglas y discernir en cada momento ese límite infranqueable de no intervención del mercado, sin importar las consecuencias para la misma vida humana que pretende desde la biopolítica fomentar. En esto, Hayek ha sido muy lúcido al declarar que en el Estado con una sociedad libre "las únicas reglas morales son las que llevan al cálculo de vidas" (Citado en Hinkelammert, 2007, p. 77). La lógica de la razón instrumental del mercado costo/beneficio cruza todas las respuestas estatales.

El liberalismo político y económico deriva en una nueva gubernamentabilidad liberal en la que hay una tensión en la relación mercado-Estado que se resuelve en la práctica de la preeminencia del mercado por sobre la razón gubernamental:

Intervencionismo significa que el gobierno quiere hacer más. Quiere interferir en los fenómenos del mercado. Si alguien protesta y dice que el gobierno no debería interferir con los negocios, la gente contesta muy a menudo: "Pero el gobierno interfiere necesariamente siempre. Si hay policías en la calle, el gobierno interfiere. Interfiere con un ladrón robando una tienda o impide que ningún hombre robe un coche". Pero cuando se trata el intervencionismo y se define qué se quiere decir con intervencionismo, estamos hablando de interferencia del gobierno en el mercado. (El que se espere que el gobierno y la policía protejan a los ciudadanos, lo que incluye a los empresarios y, por supuesto, a sus empleados, contra ataques de bandoleros nacionales o extranjeros, es en realidad una expectativa normal y necesaria para cualquier gobierno. Esa protección no es una intervención, pues la única función legítima del gobierno es precisamente producir seguridad). Lo que tenemos en mente cuando hablamos de intervencionismo es el deseo del gobierno de hacer más que impedir atracos y fraudes. Intervencionismo significa que el gobierno no solo no protege el funcionamiento eludido de la economía de mercado, sino que interfiere en los distintos fenómenos del mercado: interfiere en precios, en niveles salariales, en tipos de interés y en beneficios. (Von Mises, 1979, p. 39-40)

Parece que podemos formular la cuestión si desde el racismo de
Estado viene aparejado necesariamente un racismo económico. Para 
Foucault (2010,p.225) no haytal cosa, sus privilegios, el racismo no hace él afirma que al adversario: "cuando falta". Sin embargo, Foucault mismo se trata simplemente de eliminarlo reconoce que el nuevo orden global económicamente, de hacerle perder obliga a:

1 - El marcaje o localización de zonas vulnerables, áreas en las que el Estado no quiere que suceda nada 2 - Relajamiento y tolerancia relativa, en el cuerpo social, de delincuencia, ilegalidad, irregularidad y así se hace una regularidad, una normalidad. 3 - Contar con buen sistema de información general que permita al Estado controlar a distancia las zonas vulnerables. El objetivo no es vigilar, es la posibilidad de intervenir donde haya amenaza o eminente peligro para el poder del predominante. 4 - Construcción de consenso social a través de mass media. (Cf. Foucault, 1991, p. 165-66)

En otras palabras, el nuevo que no existe el racismo económico, orden global o globalización marca incluso hay autores que sugieren que y segrega, excluye y, por tanto, nos la biopolítica implica una necroecoalejamos de la idea foucaultiana de nomía, tal como lo dice Mbembe:

Son dos cosas. La "necropolítica" está en conexión con el concepto de "necroeconomía". Hablamos de necroeconomía en el sentido de que una de las funciones del capitalismo actual es producir a gran escala una población superflua. Una población que el capitalismo ya no tiene necesidad de explotar, pero hay que gestionar de algún modo. Una manera de disponer de estos excedentes de población es exponerlos a todo tipo de peligros y riesgos, a menudo mortales. Otra técnica consistiría en aislarlos y encerrarlos en zonas de control. Es la práctica de la "zonificación". (Varela, Lapuente Tiana y Fernández-Savater, 2016)

Verificar empíricamente lo anterior es relativamente fácil. Un par de datos: cuando uno analiza, observa la indiferencia de los países del norte hacia los problemas de los países del sur, realmente asusta la indolencia de las élites económicas y políticas ante el dolor de las víctimas en el tercer mundo. Por otra parte, el actual modelo comercial se basa en mantener un esquema perverso de intercambio desigual; les damos mano de obra barata y materia prima a bajo costo del sur, luego nosotros mismos compramos mercancías terminadas a altos 
precios (Manos Unidas, 2016). Cómo no va a existir el racismo económico si, por ejemplo, en Honduras, un multimillonario ingresa anualmente 16,460 veces lo que gana una persona situada en el $20 \%$ más pobre de la población. En promedio, el ingreso anual de los multimillonarios latino-caribeños es 4,846 veces mayor que el ingreso de los más pobres en la región (Oxfam International, 30 de septiembre de 2015).

\title{
2. Del Estado de excepción al campo de concentración en G. Agamben
}

\begin{abstract}
La tradición de los oprimidos nos enseña que el "estado de excepción" en que ahora vivimos es en verdad la regla (Benjamin, 2008, p. 309).
\end{abstract}

Agamben es un pensador italiano. funcionamiento del poder y la orgaEntre su investigación encontramos nización social, partiendo de dos una titulada Homo Sacer que trata paradigmas extremos: "el campo de sobre el pensamiento político concentración" y "el estado de excepcontemporáneo, en ella analiza el ción”. Este último se presenta como:

La forma legal de lo que no puede tener forma legal. La excepción es el dispositivo original en virtud del cual el derecho se refiere a la vida y la incluye en él por medio de la propia suspensión. (Agamben, 2010, p.10)

La importancia de este dispositivo es lo que liga al viviente con el derecho, al mismo tiempo que le abandona a él.

Según Agamben (2010), el estado de excepción es una "tierra de nadie, entre el derecho público y el hecho político". Para este autor, la creación deliberada del estado de excepción es una práctica esencial de los Estados contemporáneos. Tiene un significado biopolítico desde el momento en que la vida humana es afectada. De acuerdo a Agamben (2010), el sistema jurídico vigente desde la Primera Guerra Mundial es el estado de excepción:

[...] ha llegado a alcanzar ahora su máximo despliegue planetario. El aspecto normativo del derecho puede ser así impunemente cancelado y contrariado por una violencia gubernamental que - ignorando en el exterior el derecho internacional 
y produciendo en el interior un estado de excepción permanente - pretende, no obstante, seguir aplicando todavía un derecho. (p. 126)

Para Agamben (2010), una clara aplicación del estado de excepción es la figura de "detención indefinida de los no-ciudadanos sospechosos de actividades terroristas. Ni prisioneros ni acusados, tan solo 'detenidos' (detainees)" (p. 12-13). ¿Acaso no es ésta la situación de miles de migrantes en los centros de detención tanto en Estados Unidos como en varios países europeos? ¿Qué pasa con los solicitantes de asilo o refugio? ¿Cuál es la condición jurídica de ellos mientras se resuelve

su caso? ¿Qué pasa con las deportaciones masivas hacia lugares donde impera la violencia y la vida humana es amenazada? ¿Cuál es la condición de los migrantes desaparecidos y/o masacrados en los países de tránsito? Si una persona logra quedarse, de alguna manera, dentro de un territorio que no es donde ha nacido ¿vivirá en su condición de deportabilidad permanente? Justamente, dice Agamben, el campo de concentración y no la ciudad será nuestro espacio de convivencia:

La esencia del campo de concentración consiste en la materialización del estado de excepción y en la consiguiente creación de un espacio en el que la nuda vida y la norma entran en un umbral de indistinción [...] tal campo de concentración es pues, el estadio de Bari, en el que en 1991 la policía italiana amontonó provisionalmente a los emigrantes clandestinos albaneses antes de reexpedirlos a su país, como el velódromo de invierno en que las autoridades de Vichy agruparon a los judíos antes de entregarlos a los alemanes. (Agamben, 2006, p. 221-22)

La actual crisis humanitaria de migrantes generada por la indolencia de los Estados de origen, tránsito y destino, requiere una respuesta política y jurídica innovadora; la desfasada respuesta del racismo del Estado y el no reconocimiento de los derechos humanos de los migrantes porque se les coloca en un estado de excepción sólo agrava la crisis. Esta situación revela que la categoría política del
Estado-nación conformada aquellos que tuvieron ahí su nacimiento (la nuda vida) que se inscribe o afilia como miembro de esa nación ha colapsado, en tanto que el campo de concentración es el nuevo regulador oculto de las nuevas vidas o por lo menos, dice Agamben (2006): 
El campo de concentración es el signo de la imposibilidad de que el sistema funcione sin transformarse en una máquina letal [...] La creciente desconexión entre el nacimiento - la nuda vida - y el Estado-nación es el hecho nuevo de la política de nuestro tiempo. (p. 223)

Un ejemplo claro de esta desco- expresidente francés y líder de los nexión la tenemos por boca del republicanos, Nicolás Sarkozy:

El problema de Calais comienza en Ventimiglia, (pequeña localidad fronteriza con Italia, foco de tensiones entre los dos paises por la gestión de los migrantes) "No se trata de un problema de solidaridad, si continuamos dejando que entren en nuestro pais todos aquellos que quieren entrar, sin control, ¿cómo quieren que la situación en Calais mejore? [...] Aquellos que dicen que vamos a vaciar la jungla para enviar a toda esa gente a otras regiones, solo quieren que pase la etapa electoral, pero en 6 meses la situación será peor. Así que, lo primero que tenemos que hacer es restablecer las fronteras hasta que no haya un nuevo Schengen". (Maldonado, 26 de septiembre de 2016)

\section{Conclusiones}

A través del lente foucaultiano, vemos como el surgimiento y evolución de ciencias tales como la psicología, antropología cultural, la pedagogía, la sociología, la nueva ciencia política proporcionan al ser humano, en el siglo XX, nuevas tecnologías de poder que son instrumentalizadas por el poder político, nuevas técnicas, innovadoras terapias individuales y sociales que permiten sujetar al individuo recluyéndolo de tal manera que su única alternativa sea la de dejarse guiar por la racionalidad instrumental del libre mercado. Es lo que Foucault Llama biopolítica o gubernamentabilidad liberal. Sin menoscabo del análisis del poder en Foucault, hay que afirmar que los operadores de la economía representan un poder. No obstante, para Foucault, el poder no puede ser localizado en una institución o en el Estado predominantemente:

El poder debe analizarse como algo que circula o, mejor, como algo que sólo funciona en cadena. Nunca se localiza aquí o allá, nunca está en las manos de algunos, nunca se apropia como 
una riqueza o un bien [...] el poder transita por los individuos

[...] que ha constituido. (Foucault, 2010, p. 34)

A pesar de esta aclaración acerca de cómo opera el poder, la realidad nos dice que hay instancias como el Fondo Monetario Internacional, el Banco Mundial, los gobiernos de la Unión Europea, despachos presidenciales como el de la Casa Blanca; sin olvidar empresas transnacionales que siempre están aumentando su poder de incidir en la vida económica, política y social de las poblaciones del mundo entero. Es necesario decir que desde el análisis foucaultiano (Cfr. Foucault, 1979, p. 86), también cada individuo, para nuestro caso, cada migrante, cada persona refugiada es transida, atravesada por el poder, aunque no son un movimiento pero son parte de la red de poder, nuestro desafío es cómo articular una resistencia social.

Con Agamben, en cambio, partiendo desde Foucault pero yendo más allá de su análisis del poder, vemos como la inclusión de la vida biológica en los mecanismos del Estado no es novedosa. De acuerdo a Agamben, la biopolítica siempre ha formado parte de la esencia de las formas del poder político de raíz occidental. Con el Estado de excepción y la metáfora política de la ciudad en tanto que campo de concentración analiza las consecuencias contemporáneas de esa inclusión.
Vivimos una crisis de la convivencia, se han subvertido nuestras relaciones sociales, derivado de ello es casi imposible enfrentar las amenazas a nuestra supervivencia como especie, asistimos simultáneamente a la crisis de exclusión (dos sociedades contrapuestas) del medio ambiente, crisis del trabajo. Claramente, el desarrollo y expansión de la globalización que promueve todas las crisis al mismo tiempo nos impide enfrentarlas, consecuentemente la dignidad humana depende del cálculo costo/beneficio.

Ahora bien, si retomamos en su origen el concepto crisis que proviene del sánscrito, crisis viene de kir o kri, que significa purificar y limpiar. De kri viene crisol, elemento con el cual limpiamos el oro de las joyas, y acrisolar que quiere decir depurar y decantar. En este sentido, la crisis representa un proceso crítico, de depuración de lo esencial: sólo lo verdadero y sustancial queda, lo accidental y agregado desaparece. Esta es la decisión que debemos tomar, elegir entre el reconocimiento mutuo, la solidaridad humana y frente a eso, el capitalismo neoliberal, claramente ambas no conviven. Una opción es esencial mientras que la otra es accidental. 
Hay que redefinir los Estados nacionales y humanizar lo que se entiende por Estado soberano, si todas las Constituciones suponen la garantía primordial de la persona como origen y fin del Estado, y si en su evolución esta aplicación de la soberanía ha terminado no en una verdadera biopolítica de la vida sino en una necroeconomía y en una necropolítica a nuestro parecer, debido a que, siguiendo la argumentación de Agamben (Cfr. 2006, p. 222-24), un orden jurídico sin localización - el estado de excepción, en el que la ley es suspendida - corresponde ahora una localización sin orden jurídico, el campo de concentración, como espacio permanente de excepción.
El sistema político ya no ordena formas de vida y normas jurídicas en un espacio determinado, sino que alberga en su interior una localización dislocante que lo desborda, en el cual puede quedar incorporada cualquier forma de vida y cualquier norma (recordemos que la norma es el capitalismo salvaje, o delincuencial, como le llama el P. Juan Hernández Pico, s. j.), esta es la matriz oculta de la política actual y en este contexto es que este mundo convulsionado por las crisis ambientales, del trabajo y la crisis humanitaria constituyen la oportunidad para reconfigurar nuestras relaciones con la naturaleza y con los seres humanos.

\section{Referencias bibliográficas}

- Abraham, T. (1989). Los senderos de Foucault. Buenos Aires: Nueva Visión.

- Agamben, G. (2006). Homo sacer. El poder soberano y la nuda vida. Valencia. Pre-Textos.

Pre-Textos.

(2010). Estado de excepción. Homo sacer II, I. Valencia:

- Bauman, Z. (2007). Vida de consumo. México: FCE.

- Bauman,Z. (2011). Trabajo, consumismo y nuevos pobres. Barcelona: Gedisa.

- Benjamin, W. (2008). Sobre el concepto de historia. En Obras, libro I, vol. 2, pp. 305-317.

- Consejo Noruego para Refugiados Violencia generalizada en TNCA: Reporte IDMC 2016 Nota informativa publicada en sitio web. Recuperado de http://www.nrc.org.co/23-situacion-humanitaria/189-violencia-generalizada-en-tnca-reporte-idmc-2016

- Foucault, M. (1978). Vigilar y castigar. México. FCE.

- _ _ _ (1979). Microfísica del poder. Madrid: Ed. de la Piqueta. . (1991). Saber y verdad. Madrid: Ed de la Piqueta. 


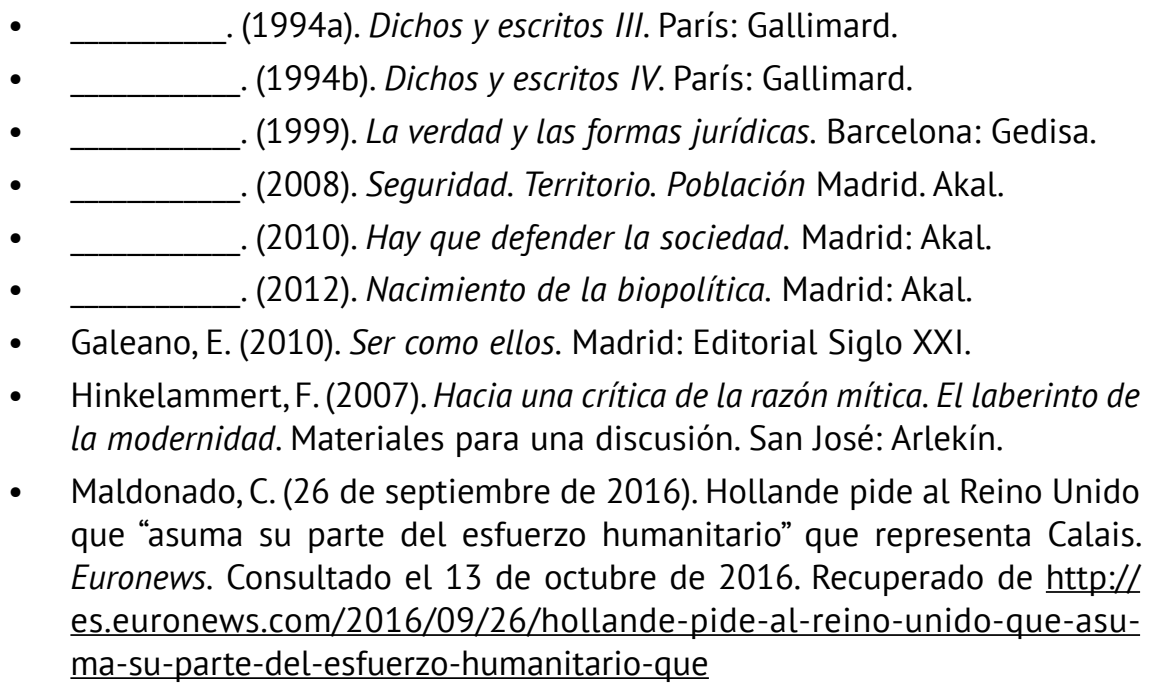

- Manos Unidas. (2016). Las causas de la pobreza en el mundo. Recuperado de http://mansunides.org/es/observatorio/causas-pobreza-en-mundo

- Oxfam International. (30 de septiembre de 2015). 200 millones de latinoamericanos en riesgo de ser pobres otra vez. Recuperado de https://www. oxfam.org/es/sala-de-prensa/notas-de-prensa/2015-09-30/200-millones-de-latinoamericanos-en-riesgo-de-ser-pobres

- Varela, A; Lapuente Tiana P. y Fernández-Savater A. (2016). Entrevista a Achille Mbembe (autor del texto: Crítica de la razón negra. Buenos Aires: Futuro anterior Ed). Recuperado de http://www.futuroanterior. com.ar/blog/cuando-el-poder-brutaliza-el-cuerpo-la-resistencia-asume-una-forma-visceral

- Von Mises, L. (1979). Economic Policy: Thoughts for Today and Tomorrow. (Traducción libre). Chicago: Ed. Regnery.

\section{Notas}

1 Disciplina y biopolítica. Si comparamos una y otra forma de poder, las podemos diferenciar como sigue: 1) En cuanto al objeto: la disciplina tiene como objeto el cuerpo individual; la biopolítica, el cuerpo múltiple, la población, el hombre como ser viviente, como perteneciente a una especie biológica. 2) En cuanto a los fenómenos considerados: mientras las disciplinas consideran los fenómenos individuales, la biopolítica estudia fenómenos de masa, en serie, de larga duración. 3) En cuanto a sus mecanismos: los 
mecanismos de las disciplinas son del orden del adiestramiento del cuerpo (vigilancia jerárquica, exámenes individuales, ejercicios repetitivos); los de la biopolítica son mecanismos de previsión, de estimación estadística, medidas globales. 4) En cuanto a la finalidad: la disciplina se propone obtener cuerpos útiles económicamente y dóciles políticamente; la biopolítica persigue el equilibrio de la población, su homeostasis, su regulación. (Cfr. Foucault, 2010, p. 213).

3 “Qué es el racismo? La capacidad de introducir un corte en el ámbito de la vida que el poder tomó a su cargo: el corte entre lo que debe vivir y lo que debe morir. En el continuum biológico de la especie humana, la aparición de las razas, su distinción, su jerarquía, la calificación de algunas como buenas y otras, al contrario, como inferiores, todo esto va a ser una manera de fragmentar el campo de lo biológico que el poder tomó a su cargo; una manera de desfasar, dentro de la población, a unos grupos con respecto de otros. [...] Por otra parte, el racismo permitirá establecer entre mi vida y la muerte del otro, una relación que no es militar y guerrera de enfrentamiento sino de tipo biológico [...] la muerte del otro, la muerte de la mala raza, de la raza inferior es lo que va a hacer que la vida en general sea más sana y más pura" (Foucault, 2010, p. 218-219). 